\title{
Standardised data reporting from pre- hospital advanced airway management - a nominal group technique update of the Utstein-style airway template
}

\author{
G. A. Sunde ${ }^{1,2,3^{*}}$ (D) A. Kottmann 1,3,4,5 J. K. Heltne ${ }^{2,6}$, M. Sandberg ${ }^{7,8}$, M. Gellerfors ${ }^{9,10,11}$, A. Krüger ${ }^{1,12}$, \\ D. Lockey ${ }^{3,13}$ and S. J. M. Sollid ${ }^{1,3,7}$
}

\begin{abstract}
Background: Pre-hospital advanced airway management with oxygenation and ventilation may be vital for managing critically ill or injured patients. To improve pre-hospital critical care and develop evidence-based guidelines, research on standardised high-quality data is important. We aimed to identify which airway data were most important to report today and to revise and update a previously reported Utstein-style airway management dataset.

Methods: We recruited sixteen international experts in pre-hospital airway management from Australia, United States of America, and Europe. We used a five-step modified nominal group technique to revise the dataset, and clinical study results from the original template were used to guide the process.

Results: The experts agreed on a key dataset of thirty-two operational variables with six additional system variables, organised in time, patient, airway management and system sections. Of the original variables, one remained unchanged, while nineteen were modified in name, category, definition or value. Sixteen new variables were added. The updated dataset covers risk factors for difficult intubation, checklist and standard operating procedure use, pre-oxygenation strategies, the use of drugs in airway management, airway currency training, developments in airway devices, airway management strategies, and patient safety issues not previously described.
\end{abstract}

Conclusions: Using a modified nominal group technique with international airway management experts, we have updated the Utstein-style dataset to report standardised data from pre-hospital advanced airway management. The dataset enables future airway management research to produce comparable high-quality data across emergency medical systems. We believe this approach will promote research and improve treatment strategies and outcomes for patients receiving pre-hospital advanced airway management.

Trial registration: The Regional Committee for Medical and Health Research Ethics in Western Norway exempted this study from ethical review (Reference: REK-Vest/2017/260).

Keywords: Airway management, Air ambulances, Emergency medical services, Intubation, Data accuracy

\footnotetext{
* Correspondence: geir.arne.sunde@norskluftambulanse.no

${ }^{1}$ Norwegian Air Ambulance Foundation, Drøbak, Norway

${ }^{2}$ Dept. of Anaesthesia and Intensive Care, Haukeland University Hospital,

Bergen, Norway

Full list of author information is available at the end of the article
}

(c) The Author(s). 2018 Open Access This article is distributed under the terms of the Creative Commons Attribution 4.0 International License (http://creativecommons.org/licenses/by/4.0/), which permits unrestricted use, distribution, and reproduction in any medium, provided you give appropriate credit to the original author(s) and the source, provide a link to the Creative Commons license, and indicate if changes were made. The Creative Commons Public Domain Dedication waiver (http://creativecommons.org/publicdomain/zero/1.0/) applies to the data made available in this article, unless otherwise stated. 


\section{Background}

Pre-hospital advanced airway management (PHAAM) with the control of oxygenation and ventilation is vital in the management of critically ill or injured patients in the field and may contribute to better outcomes [1-3]. Results from research on PHAAM are challenged by heterogeneity in provider competence, airway techniques, and the quality of data collected in many airway studies [4]. To improve pre-hospital critical care and to develop evidence-based guidelines, research based on standardised high-quality data is important $[5,6]$. Using a common and uniform set of data definitions may be the first step in such a process [7].

In pre-hospital critical care research, there has been an acceptance and tradition for using structured consensus methods to evaluate interventions, to develop guidelines, and for educational and research purposes [8, 9]. Templates for documenting and reporting of standardised data have been developed by similar methodology for out-of-hospital cardiac arrest, paediatric advanced life support, in-hospital cardiac arrest resuscitation, major incidents and disaster management, laboratory cardiopulmonary research, major trauma, emergency medical dispatch, physician staffed emergency medical services and drownings [10-19]. Developments in airway management devices, airway management strategies and training, along with patient safety issues; require that such templates are updated on a regular basis like clinical guidelines and recommendations [20].

An Utstein-style airway template was published in 2009 by an international airway expert group [21]. The feasibility of collecting standardised airway data across different patient populations and international emergency medical services (EMS) have been described [22]. The aim of this study was to update and revise the Utstein-style template for the reporting of PHAAM data, using a nominal group technique (mNGT) with international experts to identify which data variables would be most important to document today.

\section{Methods}

\section{Study design}

The revision of the Utstein-style airway template was performed using a modified nominal group technique (mNGT) consensus process, which has previously proven useful in the development of templates and guidelines for pre-hospital critical care $[9,14]$.

\section{Pre-hospital advanced airway management}

In the original template, advanced airway management was defined as the attempted insertion of an advanced airway adjunct or the administration of ventilatory assistance, in this context being "any airway management beyond manual opening of the airway and the use of simple adjuncts, such as an oropharyngeal airway". This type of management includes the use of a supraglottic airway device (SAD), tracheal intubation (TI), or emergency front of neck access (eFONA).

\section{Data variable}

A data variable should be clearly defined to avoid misinterpretation. Data points should be simple to register and possible to integrate into existing registries [21]. This requires a data variable dictionary containing information on data number, name, type of data, categories or values and definition of data variable [14].

\section{Group of experts}

The recruited experts were clinicians with leadership experience from pre-hospital critical care, had made substantial contributions to airway management research or airway management guidelines, or were considered experts in the field of PHAAM. They were recruited from networks such as European Pre-hospital Research Alliance (EUPHOREA) and the European Airway Management Society (EAMS). The experts were invited by individual email and were not aware of the composition of the group until the final consensus meeting.

\section{The modified nominal group process}

The mNGT is a systematic qualitative method involving questionnaires in repeated rounds with a final meeting aimed at consensus [9]. Our mNGT included three email rounds with questionnaires and answers (QA), and a one-day consensus meeting for plenary discussions. A fourth email round was included after the meeting for minor adjustments or comments. The results from each round were used to guide the development of the questionnaires for the following round. A third party distributed and managed the responses from the experts in individual emails and anonymised the answers. The mNGT was run from February to August 2017. The final dataset was forwarded to the experts for approval.

\section{First email round}

An Excel spreadsheet (Microsoft Corporation, Redmond, WA, USA) with the original template variables was sent to the experts (Additional file 1: Table S1). The experts were instructed to rate each variable on a 5-point Likert scale (from 1 = "totally disagree" to $5=$ "totally agree") according to how important the variable was considered to be for PHAAM and to indicate whether the original variable should be changed. The experts were then requested to suggest between three and five new variables. Additional free-text comments were allowed. These comments were not distributed to the other experts but were used to revise the variables. 


\section{Second email round}

The revised variables were organised in the original template sections, with the suggested new variables in the "optional variables section" (Additional file 2: Table S2). The experts were instructed to rank the most important variables within each section. Where relevant and for the ranked variables only, the experts indicated whether changes were warranted. Additional free-text comments were also possible in this round.

\section{Third email round}

The instructions for ranking and suggestions were similar to those of the previous round, with the revised variables now grouped in core-system, core-patient, core-postintervention, or fixed-system sections (Additional file 3: Table S3).

\section{The consensus meeting}

The aim of the consensus meeting was to finalise the variable set and discuss items that had not been cleared during the first three rounds. The main results after the email rounds were presented, and the expert comments from the preceding rounds were used to guide the discussions. The experts agreed by consensus on the changes to the template structure or variables.

\section{Ranking}

We measured expert commitment towards each variable as the number of times the individual variable was nominated by the experts. Within each section, the variables rated as "most important" received the highest score, and those rated as "least important" received the lowest score. If two variables scored equally, the variable with the highest number of individual nominations, compared to those with highest rating, was ranked higher.

\section{Results \\ Experts}

Twenty-one experts were invited to join the mNGTprocess, of whom sixteen participated in all email rounds. The experts were recruited from Australia, United States of America, and Europe. The level and type of airway experience, along with country of origin of the expert group, is described in Additional file 4. Of the experts who participated, eight attended the final consensus meeting, along with five members of the project steering group.

\section{Definition of PHAAM}

The expert group decided to keep the definition of advanced airway management unchanged from the original template, as "the attempted insertion of an advanced airway adjunct or administration of ventilatory assistance".

\section{First email round}

The experts made 127 unique suggestions for changes in variable names, categories or values in $28(44 \%)$ of the original variables. After merging similar suggestions and variables, 15 variable names and 22 categories were revised. Fifteen new variables were added to the dataset before the second email round (Additional file 2: Table S2). This process is detailed in the flowchart (Fig. 1).

\section{Second email round}

The experts indicated that change was warranted for 24 (18\%) variables and suggested 43 unique changes. Twenty-nine additional comments to improve the variables were submitted. After ranking and merging similar suggestions, 27 variables were cut, leaving 51 variables to be included in email round three. Following the experts' suggestions, the optional section was removed, and its variables were distributed in the remaining sections (Additional file 3: Table S3).

\section{Third email round}

In this round, the experts indicated a need for change in the variable name, category or definition for $23(45 \%)$ variables, and provided ten additional comments to improve the variables. The remaining variables were revised and formed the starting point for the discussions in the consensus meeting (Additional file 5: Table S4).

\section{The consensus meeting}

The experts discussed the remaining 41 variables and 10 "runner-up" variables. The experts agreed on a dataset including 32 operational variables with an additional six system variables that were identical across all missions, compared to 63 variables in the original template (Tables 1, 2, 3, 4). Of the original variables, only one variable (age) remained unchanged. Nineteen original variables were modified in terms of the variable name, category, definition or value, and the experts added 16 new variables to the revised dataset. The experts agreed on a new template structure with time, patient, airway management and system sections. The recording of PHAAM data was to cover the interval from the patient encounter on scene to when post-intervention ventilation was established, and survival to hospital (short-term survival).

\section{Discussion}

Main results

Using a modified nominal group process with international airway experts, supported by clinical study results with the original template described in the recent AIRPORT studies, we have revised the template for the reporting of standardised data from PHAAM $[22,23]$. The updated dataset includes new data points that 


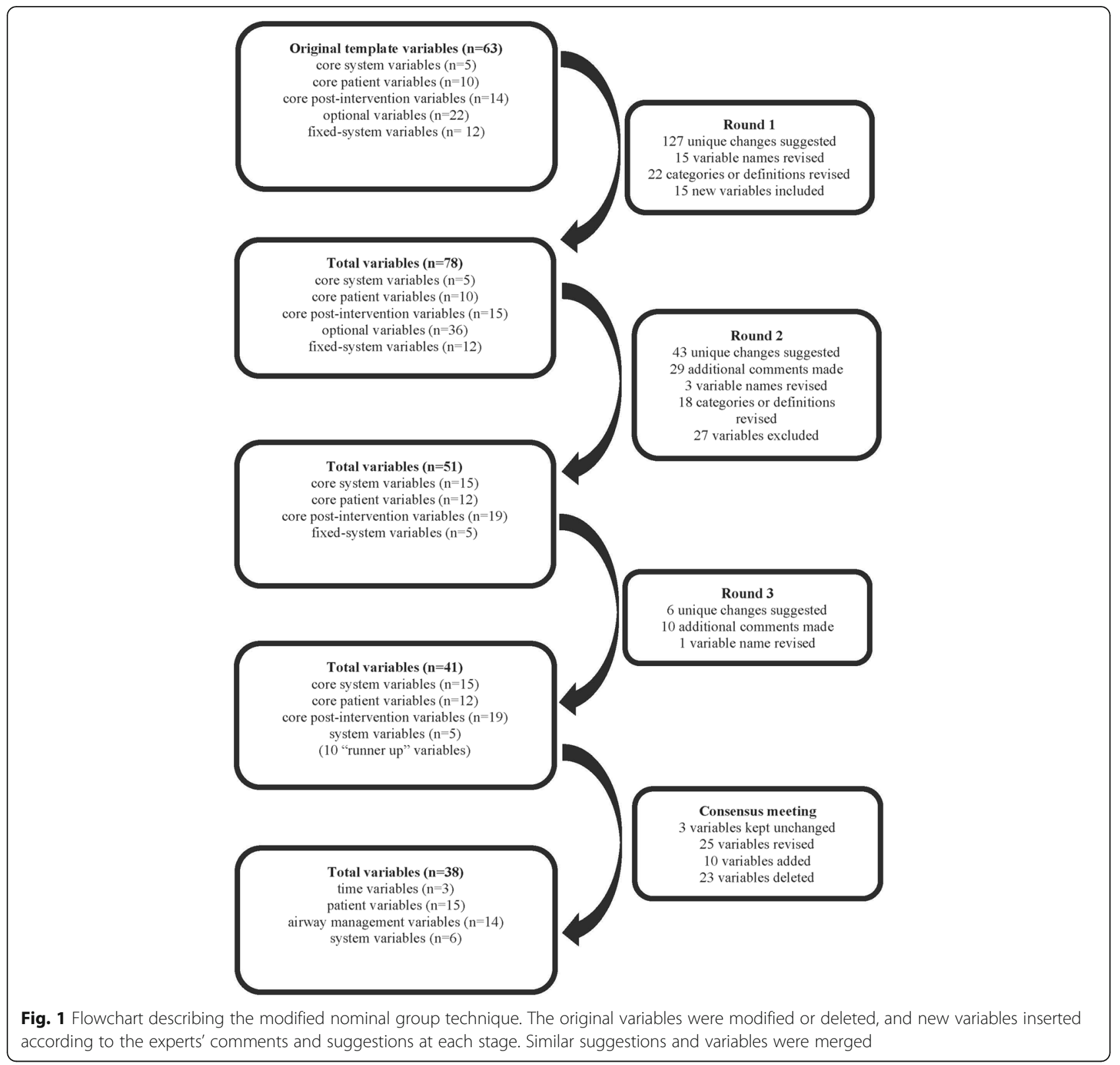

Table 1 Final time variables

\begin{tabular}{|c|c|c|c|c|}
\hline \multicolumn{5}{|c|}{ FINAL TIME VARIABLES (data provided by provider performing the intervention) } \\
\hline Number & $\begin{array}{l}\text { Data } \\
\text { variable name }\end{array}$ & $\begin{array}{l}\text { Type of } \\
\text { data }\end{array}$ & $\begin{array}{l}\text { Data variable } \\
\text { categories or values }\end{array}$ & $\begin{array}{l}\text { Definition of } \\
\text { data variable }\end{array}$ \\
\hline 1 & $\begin{array}{l}\text { Response } \\
\text { time }\end{array}$ & Continuous & Minutes & $\begin{array}{l}\text { Time from the Emergency Medical Dispatch (EMD) initiated } \\
\text { transmission of message to the EMS unit, until the time of } \\
\text { arrival of the EMS unit at the patient. }\end{array}$ \\
\hline 2 & $\begin{array}{l}\text { On-scene } \\
\text { time }\end{array}$ & Continuous & Minutes & $\begin{array}{l}\text { Time from EMS unit arrival at the patient until time of patient } \\
\text { leaving scene (or time of death if dead on scene). }\end{array}$ \\
\hline 3 & $\begin{array}{l}\text { Transport } \\
\text { time }\end{array}$ & Continuous & Minutes & $\begin{array}{l}\text { Time from patient departure from scene until patient arrival } \\
\text { at hospital. }\end{array}$ \\
\hline
\end{tabular}


Table 2 Final patient variables

\begin{tabular}{|c|c|c|c|c|}
\hline Number & $\begin{array}{l}\text { Data variable } \\
\text { name }\end{array}$ & $\begin{array}{l}\text { Type of } \\
\text { data }\end{array}$ & $\begin{array}{l}\text { Data variable categories } \\
\text { or values } \\
\text { Choose only one } \\
\text { option unless } \\
\text { otherwise stated. }\end{array}$ & Definition of data variable \\
\hline 4 & Age & Continuous & YY & $\begin{array}{l}\text { Years rounded down. } \\
\text { Ages under } 1 \text { year are reported } \\
\text { in decimals (e.g. } 6 \text { months }=0.5 \text { year) }\end{array}$ \\
\hline 5 & Gender & Nominal & $\begin{array}{l}1=\text { Female } \\
2=\text { Male } \\
3=\text { Other } / \text { Unknown }\end{array}$ & Patient gender \\
\hline 6 & $\begin{array}{l}\text { Patient } \\
\text { category }\end{array}$ & Nominal & $\begin{array}{l}1=\text { Trauma }- \text { Blunt } \\
2=\text { Trauma }- \text { Penetrating } \\
3=\text { Trauma }- \text { Head injury } \\
\text { (including TBI) } \\
4=\text { Trauma }- \text { Other } \\
\text { (including burns, strangulation, drowning, or asphyxiation) } \\
5=\text { Medical }- \text { Cardiac arrest } \\
6=\text { Medical }- \text { Respiratory distress or breathing difficulties } \\
7=\text { Medical }- \text { Intoxication } \\
8=\text { Medical }- \text { Infection (including sepsis) } \\
9=\text { Medical }- \text { Other (e.g. endocrinology } \\
\text { or other medical emergencies) } \\
10=\text { Neurology - Stroke (including cerebral } \\
\text { haemorrhage or infarction) } \\
11=\text { Neurology - Other (excluding stroke) } \\
12=\text { Psychiatry (e.g. agitation/psychosis) } \\
13=\text { Obstetrics } \\
14=\text { Other emergencies, describe: } \\
15=\text { Unknown }\end{array}$ & $\begin{array}{l}\text { Dominating reason for } \\
\text { emergency treatment } \\
\text { TBI = Traumatic Brain Injury }\end{array}$ \\
\hline 7 & $\begin{array}{l}\text { Indication for } \\
\text { airway } \\
\text { intervention }\end{array}$ & Nominal & $\begin{array}{l}1=\text { Decreased level of consciousness } \\
2=\text { Hypoxemia } \\
3=\text { Ineffective ventilation } \\
4=\text { Existing airway obstruction } \\
5=\text { Impending airway obstruction } \\
6=\text { Combative or uncooperative } \\
7=\text { Humanitarian (e.g. relief of pain or distress) } \\
8=\text { Cardiac arrest } \\
9=\text { Pre-existing airway device (e.g. SAD) } \\
\text { not working adequately } \\
10=\text { Other, describe: }\end{array}$ & $\begin{array}{l}\text { Indications for airway } \\
\text { intervention. } \\
\text { Select all that apply. }\end{array}$ \\
\hline 8 & $\begin{array}{l}\text { Patient risk } \\
\text { factors for } \\
\text { difficult } \\
\text { intubation }\end{array}$ & Nominal & $\begin{array}{l}1=\text { No risk factors for difficult intubation } \\
2=\text { Prior difficult intubation } \\
3=\text { Reduced neck mobility, } \\
\text { neck-immobilization device or } \\
\text { manual in-line stabilisation (MILS) } \\
4=\text { Severe obesity or thick/short neck } \\
5=\text { Limited mouth opening or } \\
\text { inter incisor distance }<4 \mathrm{~cm} \\
6=\text { Short Thyroid-Mental-Distance }(<6.5 \mathrm{~cm}) \\
7=\text { Significant maxillofacial or upper airway trauma } \\
8=\text { Blood, vomit, mucus or hypersalivation in airways } \\
9=\text { Pre-existing airway device (e.g. SAD) } \\
\text { not working adequately } \\
10=\text { Other, describe: } \\
11=\text { Risk factors not assessed. }\end{array}$ & $\begin{array}{l}\text { Airway assessment } \\
\text { before or during intervention } \\
\text { showing patient risk factors } \\
\text { for difficult intubation, e.g. poor } \\
\text { visualisation, foreign } \\
\text { body, blood or saliva. } \\
\text { SAD = Supraglottic } \\
\text { airway device } \\
\text { Select all that apply. }\end{array}$ \\
\hline 9 & $\begin{array}{l}\text { Aggravating conditions for } \\
\text { airway management }\end{array}$ & Nominal & $\begin{array}{l}1=\text { Patient entrapped } \\
\text { during airway management } \\
2=\text { Not } 360 \text {-degree access } \\
\text { to patient during airway management } \\
3=\text { Suboptimal provider positioning } \\
4=\text { Bright light/sunlight } \\
5=\text { Darkness } \\
6=\text { Hostile environment } \\
7=\text { In moving helicopter/ambulance }\end{array}$ & $\begin{array}{l}\text { Patient entrapped means } \\
\text { physically restrained in } \\
\text { wreckage, etc. } \\
\text { Not } 360 \text {-degree access means } \\
\text { restricted access for providers } \\
\text { to all parts of patient, e.g. cannot } \\
\text { move freely around patient or } \\
\text { patient cannot be positioned on } \\
\text { half-high stretcher for intubation. }\end{array}$ \\
\hline
\end{tabular}


Table 2 Final patient variables (Continued)

\begin{tabular}{|c|c|c|c|c|}
\hline & & & $\begin{array}{l}8=\text { In stationary } \\
\text { helicopter/ambulance } \\
9=\text { Other, describe: }\end{array}$ & $\begin{array}{l}\text { Suboptimal provider } \\
\text { positioning means suboptimal } \\
\text { intubating positioning, e.g. patient } \\
\text { flat on ground during CPR with } \\
\text { provider kneeling low or lying. } \\
\text { Hostile environment means } \\
\text { environment containing physical, } \\
\text { chemical, biological, radioactive or } \\
\text { other threats to provider safety } \\
\text { (e.g. "active shooter" scenario). } \\
\text { Select all that apply. }\end{array}$ \\
\hline 10 & Respiratory rate, initial & $\begin{array}{l}\text { Continuous } \\
\text { and } \\
\text { Nominal }\end{array}$ & $\begin{array}{l}1=\text { Number }, \text { describe } \\
2=\mathrm{NA}: \text { Did not measure } \\
3=\mathrm{NA}: \text { Could not measure }\end{array}$ & $\begin{array}{l}\text { Initial value (Baseline) } \\
\text { recorded on scene. } \\
\text { NA = Not available }\end{array}$ \\
\hline 11 & Blood pressure, initial & $\begin{array}{l}\text { Continuous } \\
\text { and } \\
\text { Nominal }\end{array}$ & $\begin{array}{l}1=\text { Number, describe } \\
\text { (Syst-BP/Dias-BP (MAP)) } \\
2=\text { NA: Did not measure } \\
3=\text { NA: Could not measure }\end{array}$ & $\begin{array}{l}\text { Initial value (Baseline) } \\
\text { recorded on scene. } \\
\text { NA = Not available }\end{array}$ \\
\hline 12 & $\mathrm{SpO}_{2}$, initial & $\begin{array}{l}\text { Continuous } \\
\text { and } \\
\text { Nominal }\end{array}$ & $\begin{array}{l}1=\text { Number }, \text { describe } \\
2=N A: \text { Did not measure } \\
3=\text { NA: Could not measure }\end{array}$ & $\begin{array}{l}\text { Initial value (Baseline) } \\
\text { recorded on scene. } \\
\text { NA = Not available }\end{array}$ \\
\hline 13 & $\begin{array}{l}\text { Blood pressure, } \\
\text { lowest prior to } \\
\text { airway management }\end{array}$ & $\begin{array}{l}\text { Continuous } \\
\text { and } \\
\text { Nominal }\end{array}$ & $\begin{array}{l}1=\text { Number, describe } \\
\text { (Syst-BP/Dias-BP (MAP)) } \\
2=\text { NA: Did not measure } \\
3=\text { NA: Could not measure }\end{array}$ & $\begin{array}{l}\text { Lowest value prior to } \\
\text { airway management } \\
\text { recorded on scene. } \\
\mathrm{NA}=\text { Not available }\end{array}$ \\
\hline 14 & $\begin{array}{l}\mathrm{SpO}_{2} \text {, lowest prior to } \\
\text { airway management }\end{array}$ & $\begin{array}{l}\text { Continuous } \\
\text { and } \\
\text { Nominal }\end{array}$ & $\begin{array}{l}1=\text { Number }, \text { describe } \\
2=\text { NA: Did not measure } \\
3=\text { NA: Could not measure }\end{array}$ & $\begin{array}{l}\text { Lowest value prior to } \\
\text { airway management } \\
\text { recorded on scene. } \\
\mathrm{NA}=\text { Not available }\end{array}$ \\
\hline 15 & $\begin{array}{l}\text { Blood pressure, } \\
\text { lowest during } \\
\text { airway management }\end{array}$ & $\begin{array}{l}\text { Continuous } \\
\text { and } \\
\text { Nominal }\end{array}$ & $\begin{array}{l}1=\text { Number, describe } \\
\text { (Syst-BP/Dias-BP (MAP)) } \\
2=\text { NA: Did not measure } \\
3=\text { NA: Could not measure }\end{array}$ & $\begin{array}{l}\text { Lowest value during } \\
\text { airway management } \\
\text { recorded on scene. } \\
\mathrm{NA}=\text { Not available }\end{array}$ \\
\hline 16 & $\begin{array}{l}\mathrm{SpO}_{2} \text {, lowest during } \\
\text { airway management }\end{array}$ & $\begin{array}{l}\text { Continuous } \\
\text { and } \\
\text { Nominal }\end{array}$ & $\begin{array}{l}1=\text { Number }, \text { describe } \\
2=\mathrm{NA}: \text { Did not measure } \\
3=\mathrm{NA}: \text { Could not measure }\end{array}$ & $\begin{array}{l}\text { Lowest value during } \\
\text { airway management } \\
\text { recorded on scene. } \\
\mathrm{NA}=\text { Not available }\end{array}$ \\
\hline 17 & $\begin{array}{l}\text { Glasgow Coma Score } \\
\text { (GCS), initial }\end{array}$ & $\begin{array}{l}\text { Continuous } \\
\text { and } \\
\text { Nominal }\end{array}$ & $\begin{array}{l}1=\text { Sum GCS } \\
\text { (Motor + Verbal + Eyes) } \\
2=\mathrm{NA}\end{array}$ & $\begin{array}{l}\text { Initial value (Baseline) } \\
\text { recorded on scene. } \\
\text { NA = Not available }\end{array}$ \\
\hline 18 & $\begin{array}{l}\text { Glasgow Coma Score } \\
\text { (GCS), lowest prior to } \\
\text { airway management }\end{array}$ & $\begin{array}{l}\text { Continuous } \\
\text { and } \\
\text { Nominal }\end{array}$ & $\begin{array}{l}1=\text { Sum GCS (Motor }+ \text { Verbal }+ \text { Eyes }) \\
2=N A\end{array}$ & $\begin{array}{l}\text { Lowest value prior to } \\
\text { airway management } \\
\text { recorded on scene. } \\
\mathrm{NA}=\text { Not available }\end{array}$ \\
\hline
\end{tabular}

reflect risk factors for difficult PHAAM, the use of checklists and standard operating procedures (SOPs), strategies for pre-oxygenation, the use of drugs in PHAAM, airway currency training, developments in airway devices, airway management strategies, and patient safety issues not previously described in the Utstein-style airway template.

\section{Time variables}

Three time intervals are important for describing a prehospital response adequately: the response time, onscene time, and patient transport time to the hospital. In comparing EMS or dispatch services across patient populations and services, these intervals are valuable for describing the EMS response, which is also closely linked to the efficiency of the dispatch process [24].

\section{Patient variables}

Patient age and sex should be included in any study population demographics [5]. We have previously shown a non-linear association between patient age and the first-attempt TI failure rates and that a significant age difference exists between trauma and non-trauma patients intubated by physician-staffed helicopter emergency medical services (HEMS), indicating that it is important to include age in a PHAAM dataset [22]. A 
Table 3 Final airway management variables

\begin{tabular}{|c|c|c|c|c|}
\hline \multicolumn{5}{|c|}{ FINAL AIRWAY MANAGEMENT VARIABLES (data provided by provider performing the intervention) } \\
\hline Number & $\begin{array}{l}\text { Data variable } \\
\text { name }\end{array}$ & $\begin{array}{l}\text { Type of } \\
\text { data }\end{array}$ & $\begin{array}{l}\text { Data variable } \\
\text { categories or values } \\
\text { Choose only one } \\
\text { option unless otherwise stated. }\end{array}$ & $\begin{array}{l}\text { Definition of } \\
\text { data variable }\end{array}$ \\
\hline 19 & $\begin{array}{l}\text { Use of checklist } \\
\text { for airway } \\
\text { management }\end{array}$ & Ordinal & $\begin{array}{l}1=\text { Written } \\
\text { checklist available } \\
\text { and used on scene } \\
2=\text { Written checklist } \\
\text { available, but not used } \\
3=\text { No checklist available }\end{array}$ & $\begin{array}{l}\text { Written checklist } \\
\text { for airway management } \\
\text { including rapid sequence } \\
\text { induction (RSI) available in } \\
\text { service and used on-scene } \\
\text { (challenge and response system). }\end{array}$ \\
\hline 20 & $\begin{array}{l}\text { Oxygenation } \\
\text { strategy for } \\
\text { airway management }\end{array}$ & Ordinal & $\begin{array}{l}1=\text { Preoxygenation with } \\
\text { non rebreathable face } \\
\text { mask before airway attempt } \\
2=\text { Preoxygenation with } \\
\text { Bag-valve-mask (BVM) } \\
\text { before airway attempt } \\
3=\text { Apnoeic oxygenation } \\
\text { during airway attempt } \\
4=\text { No preoxygenation }\end{array}$ & $\begin{array}{l}\text { Oxygenation strategies } \\
\text { used before or during } \\
\text { advanced airway management. } \\
\text { Select all that apply. }\end{array}$ \\
\hline 21 & $\begin{array}{l}\text { Sequence } \\
\text { of providers } \\
\text { performing } \\
\text { airway management }\end{array}$ & Nominal & $\begin{array}{l}\square \square \text { Emergency Medical } \\
\text { Technician } \\
\square \square \text { Paramedic } \\
\square \square \text { Nurse (non-anaesthesia) } \\
\square \square \text { Nurse (anaesthesia) } \\
\square \square \text { Physician (General practitioner } \\
\text { Or Other non-EP/ICU/ } \\
\text { Anaesthesiologist) } \\
\square \square \text { Physician (Emergency } \\
\text { Physician - EP) } \\
\square \square \text { Physician (Intensivist - ICU) } \\
\square \square \text { Physician (Anaesthesiologist) } \\
\square \square \text { Unknown }\end{array}$ & $\begin{array}{l}\text { Specify level of EMS } \\
\text { provider in sequence, } \\
\text { who performed each } \\
\text { airway management } \\
\text { attempt, numbered in } \\
\text { order of attempt. } \\
\text { Select all that apply. } \\
\text { Specify number of } \\
\text { attempt alongside } \\
\text { corresponding provider } \\
\text { with "1" and if more } \\
\text { attempts "2", "3","4". } \\
\text { E.g.: If paramedic fails } \\
\text { first attempt, then } \\
\text { physician has two } \\
\text { attempts, this is recorded as: } \\
\text { "1" Paramedic. } \\
\text { "2-3" Physician. } \\
\text { Select all that apply. }\end{array}$ \\
\hline 22 & $\begin{array}{l}\text { Sequence of airway } \\
\text { devices used for } \\
\text { airway management }\end{array}$ & Nominal & $\begin{array}{l}\square \square \text { Bag-valve-mask ventilation (BVM) } \\
\square \square \text { Supraglottic airway device with } \\
\text { suction } \\
\square \square \text { Supraglottic airway device } \\
\text { without suction } \\
\square \square \text { Direct laryngoscopy with } \\
\text { endotracheal tube } \\
\square \square \text { Direct laryngoscopy with } \\
\text { endotracheal tube and stylet } \\
\square \square \text { Direct laryngoscopy with } \\
\text { bougie and endotracheal tube } \\
\square \square \text { Video laryngoscopy } \\
\text { (Macintosh or Miller like blade) } \\
\text { with endotracheal tube } \\
\square \square \text { Video laryngoscopy } \\
\text { (Macintosh or Miller like blade) } \\
\text { with endotracheal tube and stylet } \\
\square \square \text { Video laryngoscopy } \\
\text { (Macintosh or Miller like blade) } \\
\text { with bougie and endotracheal tube } \\
\square \square \text { Video laryngoscopy } \\
\text { (hyperangulated blade) } \\
\text { with endotracheal tube } \\
\square \square \text { Video laryngoscopy } \\
\text { (hyperangulated blade) } \\
\text { with endotracheal tube and stylet }\end{array}$ & $\begin{array}{l}\text { Specify first attempt } \\
\text { with "1" and if more } \\
\text { attempts "2", "3","4". } \\
\text { E.g.: If first attempt } \\
\text { fails with endotracheal } \\
\text { intubation and direct } \\
\text { laryngoscopy, and the } \\
\text { next two attempts are } \\
\text { endotracheal intubation } \\
\text { with video laryngoscopy, } \\
\text { this is recorded as: } \\
\text { "1" Direct laryngoscopy } \\
\text { with endotracheal tube } \\
\text { "2-3" Video laryngoscopy } \\
\text { (Macintosh or Miller like blade) } \\
\text { with endotracheal tube } \\
\text { BVM = Bag-valve-mask } \\
\text { ventilation, includes } \\
\text { insertion of oro/ } \\
\text { nasopharyngeal airway. } \\
\text { If bag-valve-mask ventilation } \\
\text { prior to RSI, choose "BVM" as "1". } \\
\text { Video laryngoscopy (VL) } \\
\text { differentiates between: } \\
\text { VL with Macintosh/miller } \\
\text { like blade } \\
\text { VL with hyperangulated blade } \\
\text { Select all that apply. }\end{array}$ \\
\hline
\end{tabular}


Table 3 Final airway management variables (Continued)

FINAL AIRWAY MANAGEMENT VARIABLES (data provided by provider performing the intervention)

$\square \square$ Video laryngoscopy

(hyperangulated blade)

with bougie and endotracheal tube

$\square$ Surgical emergency

airway equipment

$\square \square$ Percutaneous emergency

airway equipment

$\square \square$ Jet-ventilation equipment

$\square \square$ Other, describe:

$\square \square$ Unknown

Airway management Ordinal results

Airway manoeuvres

following failed

airway attempt.
1 = Successful airway

management with

ET as planned

2 = Successful airway

management with

SAD as planned

3 = Successful airway

management with

surgical airway as planned

4 = Failure of primary

airway plan, and

airways secured by

alternative technique

$5=$ Final airway management

failed (loss of airways)

$6=$ Unknown

$1=$ Cricoid pressure released

$2=$ BURP/ELM manoeuvres

$3=$ Release MILS

$4=$ Reposition patient

$5=$ Ramping patient

$6=$ None $/$ Not applicable.

$1=$ None

$2=$ Thiopenta

$3=$ Ketamine

$4=$ S-ketamine

$5=$ Propofol

$6=$ Fentanyl

$7=$ Alfentanil

$8=$ Morphine

$9=$ Midazolam

$10=$ Diazepam

$11=$ Suxamethonium

$12=$ Rocuronium

$13=$ Vasopressor

$14=$ Lidocain

$15=$ Etomidate

$16=$ Other, describe:

$1=$ ET misplaced

in oesophagus AND

recognised/corrected immediately

$2=E T$ misplaced in

oesophagus and NOT

recognised/corrected immediately

$3=E T$ misplaced in left or right

main stem bronchus

$\mathrm{ET}=$ Endotracheal tube $S A D=$ Supraglottic airway device

Airway manoeuvres

following unsuccessful

airway management attempts.

BURP $=$ Backwards

upwards rightwards pressure.

$E L M=$ External laryngeal

manipulation

MILS = Manual In-line

stabilisation

Ramping $=$ The head and

trunk are elevated or

supported to align the external auditory meatus with the sternal notch

in the horizontal plane Select all that apply.

Drugs used to facilitate the actual airway intervention, not including sedation in the post-intervention

or transport phase.

Vasopressor includes any drug used as vasopressor during airway management, e.g. phenylephrine.

Local anaesthetic includes any drug used as local or regional anaesthetic, e.g. lidocaine. Select all that apply.

Complications recognised during airway management or device verification (and NOT present before the airway management). Select all that apply. $\mathrm{ET}=$ Endotracheal tube, SAD = Supraglottic airway device 
Table 3 Final airway management variables (Continued)

\begin{tabular}{|c|c|c|c|c|}
\hline \multicolumn{5}{|c|}{ FINAL AIRWAY MANAGEMENT VARIABLES (data provided by provider performing the intervention) } \\
\hline & & & $\begin{array}{l}4=\text { Incorrect positioning or } \\
\text { difficult ventilation with SAD } \\
5=\text { Dental trauma } \\
6=\text { Aspiration or } \\
\text { vomiting during } \\
\text { airway management } \\
\text { (and NOT present before) } \\
7=\text { Cardiac arrest during } \\
\text { airway management } \\
8=\text { Hypoxia during } \\
\text { airway management } \\
9=\text { Bradycardia during } \\
\text { airway management } \\
10=\text { Hypotension } \\
\text { during airway management } \\
11=\text { Complications during } \\
\text { surgical or percutaneous airway } \\
\text { (e.g. bleeding or pneumothorax) } \\
12=\text { No complications } \\
\text { (confirmed) during } \\
\text { airway management } \\
13=\text { Insufficient data } \\
\text { recording, complications unsure. }\end{array}$ & $\begin{array}{l}\text { The following definitions } \\
\text { are used:Hypoxia: Adults } \\
\text { and children: } S p O 2<90 \% \\
\text { Hypotension: } \\
\text { infants }<1 \text { year: } S B P<70 \mathrm{mmHg} \\
\text { children } 1 \text { to } 10 \text { years: } S B P<70+(2 \times \text { age }) \\
\text { children }>10 \text { years: } S B P<90 \mathrm{mmHg} \\
\text { adults: } S B P<90 \mathrm{mmHg} \text { or decrease }> \\
10 \% \text { from baseline value } \\
\text { Bradycardia } \\
\text { newborn to } 3 \text { years: } \\
<100 \text { bpm } \\
3 \text { to } 9 \text { years: }<80 \mathrm{bpm} \\
10 \text { to } 16 \text { years: }<60 \mathrm{bpm} \\
\text { adults: }<50 \mathrm{bpm} \\
\text { Select all that apply. }\end{array}$ \\
\hline 27 & $\begin{array}{l}\text { Total number } \\
\text { of successful } \\
\text { endotracheal } \\
\text { intubations the } \\
\text { provider has } \\
\text { performed } \\
\text { in patients }\end{array}$ & Ordinal & $\begin{array}{l}0=<10 \\
1=11-25 \\
2=26-50 \\
3=51-100 \\
4=101-250 \\
5=251-1000 \\
6=1001-2500 \\
7=>2500\end{array}$ & $\begin{array}{l}\text { Total number of } \\
\text { successful endotracheal } \\
\text { intubations the provider } \\
\text { has performed in patients } \\
\text { in hospital and pre-hospital } \\
\text { service, not including mannequin } \\
\text { intubations or SAD. }\end{array}$ \\
\hline 28 & $\begin{array}{l}\text { Blood pressure, } \\
\text { after finalised } \\
\text { airway management }\end{array}$ & $\begin{array}{l}\text { Continuous } \\
\text { and } \\
\text { Nominal }\end{array}$ & $\begin{array}{l}1=\text { Number, describe } \\
(\text { Syst }- \text { BP/Dias-BP (MAP)) } \\
2=\text { NA: Did not measure } \\
3=\text { NA: Could not measure }\end{array}$ & $\begin{array}{l}\text { Value recorded } \\
\text { within } 1-3 \text { min after } \\
\text { finalised airway management }\end{array}$ \\
\hline 29 & $\begin{array}{l}\mathrm{SpO}_{2} \text {, after } \\
\text { finalised airway } \\
\text { management }\end{array}$ & $\begin{array}{l}\text { Continuous } \\
\text { and } \\
\text { Nominal }\end{array}$ & $\begin{array}{l}1=\text { Number }, \text { describe } \\
2=\text { NA: Did not measure } \\
3=\text { NA: Could not measure }\end{array}$ & $\begin{array}{l}\text { Value recorded } \\
\text { within } 1-3 \text { min after } \\
\text { finalised airway management }\end{array}$ \\
\hline 30 & $\begin{array}{l}\mathrm{EtCO}_{2} \text {, after } \\
\text { finalised airway } \\
\text { management }\end{array}$ & $\begin{array}{l}\text { Continuous } \\
\text { and } \\
\text { Nominal }\end{array}$ & $\begin{array}{l}1=\text { Number }, \text { describe } \\
2=\text { NA: Did not measure } \\
3=\text { NA: Could not measure }\end{array}$ & $\begin{array}{l}\text { Value recorded } \\
\text { within } 1-3 \text { min after } \\
\text { finalised airway management }\end{array}$ \\
\hline 31 & $\begin{array}{l}\text { Ventilation, after } \\
\text { finalised airway } \\
\text { management }\end{array}$ & Nominal & $\begin{array}{l}1=\text { Spontaneous ventilation } \\
2=\text { Controlled manual ventilation } \\
3=\text { Controlled mechanical } \\
\text { ventilation (ventilator) } \\
4=\text { Mixed ventilation } \\
5=\text { Unknown }\end{array}$ & $\begin{array}{l}\text { Main mode of ventilation } \\
\text { on-scene and during } \\
\text { transport of patient } \\
\text { following finalised } \\
\text { airway management. } \\
\text { If both spontaneous } \\
\text { and controlled ventilation, } \\
\text { choose "mixed ventilation" }\end{array}$ \\
\hline 32 & Survival to hospital & Nominal & $\begin{array}{l}1=\text { Dead on-s } \\
\text { cene after ALS interventions } \\
2=\text { Alive on hospital } \\
\text { arrival (including patients being transported with } \\
\text { on-going mechanical } \\
\text { chest compressions or ECPR) } \\
3=\text { Unknown }\end{array}$ & $\begin{array}{l}\text { Patient survival status } \\
\text { limited to pre-hospital } \\
\text { treatment and arrival at } \\
\text { hospital (Short term survival) } \\
\text { ALS = Advanced Life Support } \\
\text { ECPR = extracorporeal } \\
\text { cardiopulmonary resuscitation }\end{array}$ \\
\hline
\end{tabular}

sex difference has also previously been described in emergency airway management [25].

Category describes the dominating reason for the emergency treatment, while indication describes the dominating indication for the airway intervention itself. While the patient category is among the variables most consistently reported (86\%) in airway studies, indication is less frequently reported (36\%) [5]. Describing trauma cases, differentiating between blunt trauma and penetrating trauma may be important, as 
Table 4 Final system variables

FINAL SYSTEM VARIABLES (data provided by Medical Director EMS)

\begin{tabular}{|c|c|c|c|c|}
\hline Number & $\begin{array}{l}\text { Data variable } \\
\text { name }\end{array}$ & Type of data & $\begin{array}{l}\text { Data variable } \\
\text { categories or values } \\
\text { Choose only } \\
\text { one option unless } \\
\text { otherwise stated. }\end{array}$ & Definition of data variable \\
\hline 33 & $\begin{array}{l}\text { Established } \\
\text { airway management } \\
\text { procedure (SOP) }\end{array}$ & Ordinal & $\begin{array}{l}1=\text { Yes, SOP with } \\
\text { Checklist } \\
2=\text { Yes, SOP only } \\
3=\text { No SOP }\end{array}$ & $\begin{array}{l}\text { SOP including } \\
\text { algorithm for difficult } \\
\text { intubation } \\
\text { (expected/unexpected) } \\
\text { available in EMS service. }\end{array}$ \\
\hline 34 & $\begin{array}{l}\text { Type of } \\
\text { airway currency } \\
\text { training in service }\end{array}$ & Nominal & $\begin{array}{l}1=\text { Clinical rotation } \\
\text { with regular airway } \\
\text { management practise } \\
\text { (e.g. anaesthesia) } \\
2=\text { Regular airway } \\
\text { management currency } \\
\text { assessments (e.g. RSI simulation) } \\
3=\text { Regular mannequin training } \\
4=\text { Regular cadaver training } \\
5=\text { Other, describe }\end{array}$ & $\begin{array}{l}\text { Clinical rotation: } \\
\text { describes system } \\
\text { with regular airway } \\
\text { management currency } \\
\text { (e.g. anaesthesia practise). } \\
\text { Regular airway management } \\
\text { currency assessment } \\
\text { (e.g. RSI simulation) } \\
\text { describes systems with } \\
\text { simulation or virtual } \\
\text { training for airway } \\
\text { management currency. } \\
\text { Cadaver and mannequin } \\
\text { describes systems with } \\
\text { regular airway management } \\
\text { skill training. } \\
\text { Select all that apply. }\end{array}$ \\
\hline 35 & $\begin{array}{l}\text { Type of tracheal } \\
\text { tube confirmation } \\
\text { technique used } \\
\text { in service }\end{array}$ & Nominal & $\begin{array}{l}1=\text { Auscultation only } \\
2=\text { Capnometry only } \\
3=\text { Waveform capnography } \\
4=\text { Colorimetric detector (e.g. Easycap) } \\
5=\text { Ultrasound } \\
6=\text { Other, describe: } \\
7=\text { None }\end{array}$ & $\begin{array}{l}\text { Capnometry is a } \\
\text { measurement of } \\
\mathrm{ETCO}_{2} \text { i.e., analysis alone) } \\
\text { without a continuous } \\
\text { written record or waveform. } \\
\text { Waveform capnography } \\
\text { includes waveforms of } \\
\text { inspiration and expiration } \\
\text { pattern along with } \\
\text { values for } \mathrm{ETCO}_{2} \text {. } \\
\text { Select all that apply. }\end{array}$ \\
\hline 36 & $\begin{array}{l}\text { Airway management } \\
\text { devices used in service }\end{array}$ & Nominal & $\begin{array}{l}1=\text { Bag-valve-mask ventilation } \\
2=\text { Supraglottic airway } \\
\text { device with suction } \\
3=\text { Supraglottic airway } \\
\text { device without suction } \\
4=\text { Direct laryngoscopy } \\
\text { with endotracheal tube } \\
\text { (including bougie and/or stylet). } \\
5=\text { Video laryngoscopy } \\
\text { with Macintosh or Miller } \\
\text { like blade and endotracheal } \\
\text { tube (including bougie and/or stylet). } \\
6=\text { Video laryngoscopy } \\
\text { with hyperangulated blade } \\
\text { and endotracheal tube } \\
\text { (including bougie and/or stylet). } \\
7=\text { Surgical emergency } \\
\text { airway equipment } \\
8=\text { Percutaneous } \\
\text { emergency airway equipment } \\
9=\text { Jet-ventilation equipment } \\
10=\text { Other, describe: } \\
11=\text { Unknown }\end{array}$ & $\begin{array}{l}\text { Airway devices } \\
\text { available in service } \\
\text { and provider who } \\
\text { knows how to use it. } \\
\text { Bag-valve-mask } \\
\text { ventilation includes } \\
\text { insertion of } \\
\text { oro/nasopharyngeal airway. } \\
\text { Video laryngoscopy } \\
\text { VL) differentiates between: } \\
\text { VL with Macintosh/ } \\
\text { miller like blade } \\
\text { VL with hyperangulated blade } \\
\text { Select all that apply. }\end{array}$ \\
\hline 37 & $\begin{array}{l}\text { Drugs for } \\
\text { airway management } \\
\text { available in service }\end{array}$ & Nominal & $\begin{array}{l}1=\text { None } \\
2=\text { Thiopental } \\
3=\text { Ketamine } \\
4=\text { S-ketamine }\end{array}$ & $\begin{array}{l}\text { Drugs used for airway } \\
\text { management, available } \\
\text { on scene and someone c } \\
\text { ompetent to administer them. }\end{array}$ \\
\hline
\end{tabular}


Table 4 Final system variables (Continued)

\begin{tabular}{|c|c|c|c|c|}
\hline \multicolumn{5}{|c|}{ FINAL SYSTEM VARIABLES (data provided by Medical Director EMS) } \\
\hline & & & $\begin{array}{l}5=\text { Propofol } \\
6=\text { Fentanyl } \\
7=\text { Alfentanil } \\
8=\text { Morphine } \\
9=\text { Midazolam } \\
10=\text { Diazepam } \\
11=\text { Suxamethonium } \\
12=\text { Rocuronium } \\
13=\text { Vasopressor } \\
14=\text { Lidocain } \\
15=\text { Etomidate } \\
16=\text { Other }, \text { describe: }\end{array}$ & Select all that apply. \\
\hline 38 & $\begin{array}{l}\text { Highest Level } \\
\text { of EMS provider } \\
\text { involved in airway management on-scene }\end{array}$ & Nominal & $\begin{array}{l}1=\text { Emergency } \\
\text { Medical Technician (EMT) } \\
2=\text { Paramedic } \\
3 \text { = Nurse (non-anaesthesia) } \\
4=\text { Nurse (anaesthesia) } \\
5=\text { Physician } \\
\text { (General practitioner or } \\
\text { other non-EP/ICU/Anaesthesiologist) } \\
6=\text { Physician } \\
\text { (Emergency Physician - EP) } \\
7=\text { Physician } \\
\text { (Intensivist - ICU) } \\
8=\text { Physician (Anaesthesiologist) } \\
9=\text { Unknown }\end{array}$ & $\begin{array}{l}\text { Highest level } \\
\text { of EMS provider } \\
\text { present on scene } \\
\text { and involved in } \\
\text { airway management; } \\
\text { including assessment, } \\
\text { drugs or intervention. }\end{array}$ \\
\hline
\end{tabular}

strategies for both airway management and haemorrhage control can differ between these groups [26]. Traumatic brain injury (TBI) is another major category where treatment options may differ, and airway management competence is linked to mortality in this group [27]. In non-trauma patients, the experts agreed that distinguishing between cardiac arrest; neurological emergencies; respiratory distress or breathing difficulties; intoxication; infection (including sepsis); and other medical emergencies may be important when describing PHAAM.

Airway assessment is an integral part of providing safe pre-hospital anaesthesia and advanced airway management. Patient risk factors for difficult bag-valvemask (BVM) ventilation or TI were not included in the original template. Optimal patient positioning may maximise the chance of successful PHAAM [20]. And, the pre-hospital setting contains some unique external factors, which may influence access to the patient and hence airway management success $[28,29]$. The expert panel agreed that such risk factors should be described in the dataset.

Key vital signs are commonly used to assess the physiological status of patients in many clinical settings [30]. The experts agreed that single values, not ranges of measurements, should be recorded in general. Agreeing on the necessity of an initial baseline measurement of the patient's respiratory rate (RR), blood pressure, peripheral oxygen saturation $\left(\mathrm{S}_{\mathrm{P}} \mathrm{O}_{2}\right)$ and
Glasgow coma score (GCS), the experts also found that recording the lowest value prior to and during the airway intervention was important. The recording of endtidal carbon dioxide $\left(\mathrm{ETCO}_{2}\right)$ after finalised PHAAM is important to confirm tube placement but may also be beneficial for optimising advanced life support (ALS) [31].

\section{Airway management variables}

The use of pre-TI checklists for PHAAM to reduce adverse events and improve patient safety is recommended [32]. However, a recent multicentre randomised trial of checklist use in rapid sequence intubation (RSI) found no reduction in complication rates compared to standard practice without checklists [33]. While experienced providers may rely on mental checklists, inexperienced providers may depend on written checklists in a challenge and response system. The experts agreed that the airway dataset should only document whether a written checklist is available on scene and whether the checklist was used.

The Difficult Airway Society recommends that preinduction airway plans are briefed to the team and that failure of primary or secondary airway plans are clearly declared to facilitate control of the patients' airways and to avoid complications [20]. Although a prediction of a difficult airway is not always reliable, a planned and verbalised pre-induction airway plan should be in place 
prior to an RSI [20, 34]. The experts agreed that recording whether PHAAM was successful as planned and documenting whether the final airway attempt failed were important.

There is evidence for an association between airway management skills and patient outcomes in PHAAM $[27,35]$. Poorly performed airway management carries significant mortality and morbidity risks, and adequate training and experience is important for patient safety [35]. As success and complication rates are also associated with provider competence and experience, the experts found that recording the level and sequence of providers performing the actual airway interventions was necessary [36]. Specifying the sequence of providers may provide new knowledge of PHAAM, especially where primary airway management fails and an unanticipated difficult airway in the field must be handled. Furthermore, the results from airway studies are difficult to interpret and compare without such information. The total number of successful TIs the provider has performed in patients in hospital and in pre-hospital service may be regarded as a reasonable surrogate for total airway competence [37].

Patient pre-oxygenation is standard practice during any anaesthesia induction, aiming at maximising the oxygen reserves and delaying the onset of desaturation for several minutes in the event of a failed or difficult primary airway intervention [38]. Strategies for preoxygenation have improved over the last decades, targeting both the pre-induction phase, and the apnoea time after the induction of anaesthesia [39]. Pre-oxygenation was not included in the original template; however, the experts agreed that as pre-oxygenation might have a crucial effect on avoiding hypoxia during the apnoeic phase of TI and should be recorded [38].

Although RSI is standard practice for emergency anaesthesia in patients with a risk of pulmonary aspiration, the definition of RSI may not be uniform across international EMS services [32, 40]. RSI implies a transition from full consciousness with intact airway reflexes to complete unconsciousness. Ensuring optimal TI conditions with a high first pass success rate, backed up by rehearsed airway plans should the primary TI attempts fail, is important [20,34]. Although the use of NMBA may increase TI success rates, a setting where the patient is rendered apnoeic may be challenging if primary airway management fails [36].

The most critical part of PHAAM may be the airway intervention itself, especially when performed during suboptimal conditions in the field [41]. Limiting the number of attempts is recommended, before declaring failed TI and proceeding with an alternative airway device [20]. Thus, specifying the number of attempts and type of device used in each attempt in sequential order may be important when documenting airway complications as an integral part of a patient safety culture. Rescue manoeuvres, such as backwards-upwards-rightwards-pressure or external laryngeal manipulation are manoeuvres commonly used to optimise TI conditions [34].This was not included in the original template, but the experts found that describing these manoeuvres in the revised dataset was useful.

In recent years, video laryngoscopy (VL) has been increasingly used in airway management [42, 43]. Although VL may improve the glottic view and be beneficial in the context of a difficult airway, little evidence exists today showing that VL reduces the number of TI attempts or airway complication rates, compared to direct laryngoscopy (DL) $[44,45]$. As a technique involving an airway device, VL was not included in the original template. The benefit of VL in PHAAM still needs to be demonstrated, therefore the main types of $\mathrm{VL}$ and DL were included in the revised dataset.

The possibility of isolating different generic drugs used for PHAAM across patient categories might provide new knowledge, and the experts agreed to include the most common generic drugs used in PHAAM today.

The experts agreed that survival to hospital (shortterm survival) should be recorded. Additionally, "dead on arrival" implies that no ALS procedures have been provided and should not be included. Rather, "dead onscene after ALS interventions" or "alive on hospital arrival" should be documented. This categorisation includes patients being transported to the hospital with on-going mechanical chest compressions or extracorporeal cardiopulmonary resuscitation (ECPR) [46].

\section{System variables}

SOPs, including algorithms for unexpected difficult airway management, are emerging as an indispensable part of patient safety and quality systems [32]. The experts agreed that recording whether airway management SOPs are available in the individual EMS is important, also recognising the importance of developing robust clinical governance systems for pre-hospital critical care [47]. The experts agreed that the types of recurring airway management training provided by the EMS systems are important to record.

\section{Value of standardised data}

To be able to compare interventions or level of care across systems, standardized research data using common terminology, data definitions or quality indicators are required [48]. Developing common variables and definitions is an on-going process and it is important to identify the correct variables to use in airway research projects and for benchmarking of airway management across EMS [14]. The results from consensus processes such as ours are not the endpoint, as dissemination and 
implementation of the results into clinical service are the final aims [49]. A few EMS have successfully implemented the original template into clinical service; however, endorsement by internationally recognised airway societies, research groups, or key EMS stakeholders, along with interoperable health information systems, may be vital to secure a broad implantation of the airway template in international EMS $[8,50,51]$.

The feasibility of collecting airway and trauma data using standardised templates have been shown, and it is important that data in comparative research projects are collected in a uniform manner $[22,48,52]$. To date, twenty-two articles have described data collection methods adhering to, or adapted to, the original Utsteinstyle airway template $[3,5,8,21-23,50,52-66]$. A further twenty-three articles have referred to the original publication $[6,8,13,16,35,36,41,67-82]$. However, as technology evolves, the availability of new possibilities of data capture from devices like video or body cameras, or streaming of monitor data directly to hospital data systems, may influence this kind of research [83, 84]. Therefore, how study data are obtained may be important to document to increase accuracy of data.

\section{Limitations}

The scientific value of consensus methods, such as mNGT or the Delphi surveys, have been questioned, and no method is considered a "gold standard" [85]. Nonetheless, consensus methods are useful tools to assess agreement on questions for which hard evidence is difficult to obtain. We believe that recruiting a broad panel of experts according to predefined criteria, from fourteen countries across Europe, Australia and United States of America, may have reduced a possible selection bias and yielded a representative list of variables with scientific value. Keeping the preliminary email rounds and proposals anonymous from round one to three was important to avoid the influence of "loud-speaking" experts and to reduce the effect of a strong reputation or opinion on other more "silent-speaking" experts [9]. Each QA round was handled confidentially so that the experts were not aware of the answers or comments from the other experts.

\section{Conclusions}

Using a mNGT with international experts, we have updated the dataset to report standardised data from prehospital advanced airway management. The dataset enables future airway management research to produce comparable high-quality data across emergency medical systems. We believe this approach will promote research and improve treatment strategies and outcomes for patients receiving pre-hospital advanced airway management.'

\section{Additional files}

Additional file 1: Questionnaire first email round. (XLSX $24 \mathrm{~kb})$

Additional file 2: Questionnaire second email round. (XLSX $21 \mathrm{~kb}$ )

Additional file 3: Questionnaire third email round. (XLSX $20 \mathrm{~kb}$ )

Additional file 4: Expert group composition. (DOCX $140 \mathrm{~kb}$ )

Additional file 5: Questionnaire consensus meeting. (XLSX $17 \mathrm{~kb}$ )

\section{Abbreviations}

ALS: Advanced life support; BVM: Bag-valve-mask; DL: Direct laryngoscopy; ECPR: Extracorporeal cardiopulmonary resuscitation; eFONA: Emergency front of neck access; EMS: Emergency medical services; $\mathrm{ETCO}_{2}$ : End-tidal carbon dioxide; GCS: Glasgow coma score; HEMS: Helicopter emergency medical services; mNGT: Modified nominal group technique; NMBA: Neuromuscular blocking agents; PHAAM: Pre-hospital advanced airway management; QA: Questionnaires and answers; RR: Respiratory rate; RSI: Rapid sequence intubation; SAD: Supraglottic airway device; SOP: Standard operating procedure; $\mathrm{S}_{\mathrm{P}} \mathrm{O}_{2}$ : Peripheral oxygen saturation; TBI: Traumatic brain injury; TI: Tracheal intubation; VL: Video laryngoscopy

\section{Acknowledgements}

Our sincerest thanks go to the international airway expert group who made this study possible: Ilkka Virkkunen, Wolfgang Voelckel, Peter Paal, Lorenz Theiler, Massimiliano Sorbello, Richard Lyon, Kate Crewdson, Brian Burns, Leif Rognås, Björn Hossfeld, Alasdair Corfield, Daniel Davis, Tomasz Gaszyński, and Pavel Michalek. We extend a special gratitude to international research coordinator Kirsti Strømmen Holm at the Norwegian Air Ambulance Foundation for her invaluable work in coordinating all QAs in the email rounds with the experts and arranging the consensus group meeting. Lastly, we extend our appreciation to the Lærdal Foundation for their support towards the consensus meeting of the international airway experts.

\section{Funding}

Financial support and sponsorship: The Norwegian Air Ambulance Foundation funds the part time PhD-grant (50\%) for GAS, the full time PhDgrant for AK, and is the main funding source of the academic positions for two of the supervisors (JKH and SJMS). The SICPA Foundation granted AK for a research fellowship in Norway. The participating experts received no financial support for their participation in this study. A grant of 75000 NOK (Grant 3341) was awarded from the Lærdal Foundation towards two projects: one revising the airway template and one developing quality indicators for pre-hospital airway management. The grant contributed to the accomplishment of the consensus meeting of experts. The Norwegian Air Ambulance Foundation and the Lærdal Foundation, however, had no authority in any part of the study design, project management, data collection, data analysis or interpretation, writing of the manuscript or publication.

\section{Availability of data and materials \\ The datasets generated during this study are included in this published article.}

\section{Authors' contributions}

GAS conceived the study, designed the study protocol, was responsible for the ethical applications in Norway, developed the questionnaires for the consensus rounds, contributed to the data collection and analysis, and wrote the first and final drafts of the manuscript. AK conceived the study, designed the study protocol, developed the questionnaires for the consensus rounds, contributed to the data collection and analysis, and helped draft the manuscript. JKH aided in the design of the study protocol, contributed to the data collection and analysis, and helped draft the manuscript. MS contributed to the data collection and analysis and helped draft the manuscript. MG contributed to the data collection and analysis and helped draft the manuscript. AKR aided in the design of the study protocol, contributed to the data collection and analysis, and helped draft the manuscript. DL aided in the design of study and the drafting of the manuscript. SJMS aided in the design of the study protocol, contributed to the data collection and analysis, moderated the consensus meeting, and 
helped draft the manuscript. All authors read and approved the final manuscript.

\section{Ethics approval and consent to participate}

Not applicable. The Regional Committee for Medical and Health Research Ethics in Western Norway exempted this study from ethical review as it did not include human research data (Reference: REK-Vest/2017/260)

\section{Competing interests}

The authors declare that they have no competing interests.

\section{Publisher's Note}

Springer Nature remains neutral with regard to jurisdictional claims in published maps and institutional affiliations.

\section{Author details}

${ }^{1}$ Norwegian Air Ambulance Foundation, Drøbak, Norway. ${ }^{2}$ Dept. of Anaesthesia and Intensive Care, Haukeland University Hospital, Bergen, Norway. ${ }^{3}$ Faculty of Health Sciences, University of Stavanger, Stavanger, Norway. ${ }^{4}$ Emergency Dept., University Hospital of Lausanne, Lausanne, Switzerland. ${ }^{5}$ Swiss Air Ambulance - Rega, Zürich, Switzerland. ${ }^{6}$ Dept. of Medical Sciences, University of Bergen, Bergen, Norway. ${ }^{7}$ Air Ambulance Dept., Oslo University Hospital, Oslo, Norway. ${ }^{8}$ Faculty of Medicine, University of Oslo, Oslo, Norway. ${ }^{9}$ Karolinska Institutet, Dept. of Clinical Science and Education, Section of Anaesthesiology and Intensive Care, Stockholm, Sweden. ${ }^{10}$ Swedish Air Ambulance (SLA), Mora, Sweden. ${ }^{11}$ Dept. of Anaesthesiology and Intensive Care, Södersjukhuset, Stockholm, Sweden. ${ }^{12}$ Dept. of Emergency Medicine and Pre-hospital Services, St. Olavs Hospital, Trondheim, Norway. ${ }^{13}$ London's Air Ambulance, Bartshealth NHS Trust, London, UK.

\section{Received: 9 February 2018 Accepted: 9 May 2018}

\section{Published online: 04 June 2018}

\section{References}

1. Nolan JP, Deakin CD, Soar J, Bottiger BW, Smith G. European resuscitation council guidelines for resuscitation 2005. Section 4. Adult advanced life support. Resuscitation. 2005;67(Suppl 1):S39-86.

2. Eich C, Roessler M, Nemeth M, Russo SG, Heuer JF, Timmermann A. Characteristics and outcome of prehospital paediatric tracheal intubation attended by anaesthesia-trained emergency physicians. Resuscitation. 2009; 80:1371-7.

3. Lockey DJ, Crewdson K, Davies G, Jenkins B, Klein J, Laird C, et al. AAGBI: safer pre-hospital anaesthesia 2017: Association of Anaesthetists of Great Britain and Ireland. Anaesthesia. 2017;72:379-90.

4. Fevang E, Perkins Z, Lockey D, Jeppesen E, Lossius HM. A systematic review and meta-analysis comparing mortality in pre-hospital tracheal intubation to emergency department intubation in trauma patients. Crit Care. 2017:21:192

5. Lossius HM, Sollid SJ, Rehn M, Lockey DJ. Revisiting the value of pre-hospital tracheal intubation: an all time systematic literature review extracting the Utstein airway core variables. Crit Care. 2011;15:R26.

6. Davis DP. The need for standardized data reporting for prehospital airway management. Crit Care. 2011;15:133.

7. Coats TJ. A revised Utstein template for trauma. Emerg Med J. 2010;27:339.

8. Lossius HM, Kruger AJ, Ringdal KG, Sollid SJ, Lockey DJ. Developing templates for uniform data documentation and reporting in critical care using a modified nominal group technique. Scand J Trauma Resusc Emerg Med. 2013;21:80

9. Murphy MK, Black NA, Lamping DL, McKee CM, Sanderson CF, Askham J, et al. Consensus development methods, and their use in clinical guideline development. Health Technol Assess. 1998;2(i-iv):1-88.

10. Castren M, Karlsten R, Lippert F, Christensen EF, Bovim E, Kvam AM, et al. Recommended guidelines for reporting on emergency medical dispatch when conducting research in emergency medicine: the Utstein style. Resuscitation. 2008;79:193-7.

11. Cummins RO, Chamberlain D, Hazinski MF, Nadkarni V, Kloeck W, Kramer E, et al. Recommended guidelines for reviewing, reporting, and conducting research on in-hospital resuscitation: the in-hospital 'Utstein style'. American Heart Association Circulation. 1997;95:2213-39.
12. Idris AH, Becker LB, Ornato JP, Hedges JR, Bircher NG, Chandra NC, et al. Utstein-style guidelines for uniform reporting of laboratory CPR research. A statement for healthcare professionals from a task force of the American Heart Association, the American College of Emergency Physicians, the American College of Cardiology, the European resuscitation council, the Heart and Stroke Foundation of Canada, the Institute of Critical Care Medicine, the Safar Center for Resuscitation Research, and the Society for Academic Emergency Medicine. Writing group. Circulation. 1996;94:2324-36.

13. Kruger AJ, Lockey D, Kurola J, Di Bartolomeo S, Castren M, Mikkelsen S, et al. A consensus-based template for documenting and reporting in physicianstaffed pre-hospital services. Scand J Trauma Resusc Emerg Med. 2011;19:71.

14. Ringdal KG, Coats TJ, Lefering R, Di Bartolomeo S, Steen PA, Roise O, et al. The Utstein template for uniform reporting of data following major trauma: a joint revision by SCANTEM, TARN, DGU-TR and RITG. Scand J Trauma Resusc Emerg Med. 2008;16:7.

15. Dick WF, Baskett PJ. Recommendations for uniform reporting of data following major trauma-the Utstein style. A report of a working party of the international trauma Anaesthesia and critical care society (ITACCS). Resuscitation. 1999:42:81-100.

16. Fattah S, Rehn M, Lockey D, Thompson J, Lossius HM, Wisborg T, et al. A consensus based template for reporting of pre-hospital major incident medical management. Scand J Trauma Resusc Emerg Med. 2014:22:5.

17. Idris AH, Berg RA, Bierens J, Bossaert L, Branche CM, Gabrielli A, et al. Recommended guidelines for uniform reporting of data from drowning: the "Utstein style". Circulation. 2003;108:2565-74.

18. Cummins RO, Chamberlain DA, Abramson NS, Allen M, Baskett PJ, Becker L, et al. Recommended guidelines for uniform reporting of data from out-ofhospital cardiac arrest: the Utstein style. A statement for health professionals from a task force of the American Heart Association, the European resuscitation council, the Heart and Stroke Foundation of Canada, and the Australian resuscitation council. Circulation. 1991:84:960-75.

19. Zaritsky A, Nadkarni V, Hazinski MF, Foltin G, Quan L, Wright J, et al. Recommended guidelines for uniform reporting of pediatric advanced life support: the pediatric Utstein style. A statement for healthcare professionals from a task force of the American Academy of Pediatrics, the American Heart Association, and the European resuscitation council. Resuscitation. 1995:30:95-115.

20. Frerk C, Mitchell VS, McNarry AF, Mendonca C, Bhagrath R, Patel A, et al. Difficult airway society 2015 guidelines for management of unanticipated difficult intubation in adults. Br J Anaesth. 2015;115:827-48.

21. Sollid SJ, Lockey D, Lossius HM. Pre-hospital advanced airway management expert g. A consensus-based template for uniform reporting of data from pre-hospital advanced airway management. Scand J Trauma Resusc Emerg Med. 2009:17:58.

22. Sunde GA, Heltne JK, Lockey D, Burns B, Sandberg M, Fredriksen K, et al. Airway management by physician-staffed helicopter emergency medical services - a prospective, multicentre, observational study of 2,327 patients. Scand J Trauma Resusc Emerg Med. 2015:23:57.

23. Sunde GA, Sandberg M, Lyon R, Fredriksen K, Burns B, Hufthammer KO, et al. Hypoxia and hypotension in patients intubated by physician staffed helicopter emergency medical services - a prospective observational multiCentre study. BMC Emerg Med. 2017;17:22.

24. Castrén M, Bohm K, Kvam AM, Bovim E, Christensen EF, Steen-Hansen JE, et al. Reporting of data from out-of-hospital cardiac arrest has to involve emergency medical dispatching - taking the recommendations on reporting OHCA the Utstein style a step further. Resuscitation. 2011;82:1496-500.

25. Cook TM, Woodall N, Frerk C, Fourth National Audit P. major complications of airway management in the UK: results of the fourth National Audit Project of the Royal College of Anaesthetists and the difficult airway society. Part 1: anaesthesia. Br J Anaesth. 2011;106:617-31.

26. Spahn DR, Bouillon B, Cerny V, Coats TJ, Duranteau J, Fernandez-Mondejar E, et al. Management of bleeding and coagulopathy following major trauma: an updated European guideline. Crit Care. 2013;17:R76.

27. Bossers SM, Schwarte LA, Loer SA, Twisk JW, Boer C, Schober P. Experience in prehospital endotracheal intubation significantly influences mortality of patients with severe traumatic brain injury: a systematic review and metaanalysis. PLoS One. 2015;10:e0141034.

28. Helm M, Hossfeld B, Schafer S, Hoitz J, Lampl L. Factors influencing emergency intubation in the pre-hospital setting-a multicentre study in the German helicopter emergency medical service. Br J Anaesth. 2006;96:67-71. 
29. Combes X, Jabre P, Jbeili C, Leroux B, Bastuji-Garin S, Margenet A, et al. Prehospital standardization of medical airway management: incidence and risk factors of difficult airway. Acad Emerg Med. 2006;13:828-34.

30. Fleming S, Thompson M, Stevens R, Heneghan C, Pluddemann A, Maconochie I, et al. Normal ranges of heart rate and respiratory rate in children from birth to 18 years of age: a systematic review of observational studies. Lancet. 2011;377:1011-8.

31. Heradstveit BE, Sunde K, Sunde GA, Wentzel-Larsen T, Heltne JK. Factors complicating interpretation of capnography during advanced life support in cardiac arrest-a clinical retrospective study in 575 patients. Resuscitation. 2012;83:813-8.

32. Sherren PB, Tricklebank S, Glover G. Development of a standard operating procedure and checklist for rapid sequence induction in the critically ill. Scand J Trauma Resusc Emerg Med. 2014;22:41.

33. Janz DR, Semler MW, Joffe AM, Casey JD, Lentz RJ, deBoisblanc BP, et al. A multicenter randomized trial of a checklist for endotracheal intubation of critically ill adults. Chest. 2018;153:816-24.

34. Henderson JJ, Popat MT, Latto IP, Pearce AC. Difficult airway society guidelines for management of the unanticipated difficult intubation. Anaesthesia. 2004;59:675-94.

35. Crewdson K, Lockey DJ, Roislien J, Lossius HM, Rehn M. The success of prehospital tracheal intubation by different pre-hospital providers: a systematic literature review and meta-analysis. Crit Care. 2017;21:31.

36. Lockey DJ, Crewdson K, Lossius HM. Pre-hospital anaesthesia: the same but different. Br J Anaesth. 2014;113:211-9.

37. Konrad C, Schupfer G, Wietlisbach M, Gerber H. Learning manual skills in anesthesiology: is there a recommended number of cases for anesthetic procedures? Anesth Analg. 1998;86:635-9.

38. Bouroche G, Bourgain JL. Preoxygenation and general anesthesia: a review. Minerva Anestesiol. 2015:81:910-20.

39. McNarry AF, Patel A. The evolution of airway management - new concepts and conflicts with traditional practice. Br J Anaesth. 2017;119:1154-i66.

40. Koerber JP, Roberts GE, Whitaker R, Thorpe CM. Variation in rapid sequence induction techniques: current practice in Wales. Anaesthesia. 2009;64:54-9.

41. Burns B, Habig K, Eason H, Ware S. Difficult intubation factors in prehospital rapid sequence intubation by an Australian helicopter emergency medical service. Air Med J. 2016;35:28-32.

42. Cavus E, Callies A, Doerges V, Heller G, Merz S, Rosch P, et al. The C-MAC videolaryngoscope for prehospital emergency intubation: a prospective, multicentre, observational study. Emerg Med J. 2011;28:650-3.

43. Mosier J, Chiu S, Patanwala AE, Sakles JC. A comparison of the GlideScope video laryngoscope to the C-MAC video laryngoscope for intubation in the emergency department. Ann Emerg Med. 2013;61:414-20 e1.

44. Lewis SR, Butler AR, Parker J, Cook TM, Schofield-Robinson OJ, Smith AF. Videolaryngoscopy versus direct laryngoscopy for adult patients requiring tracheal intubation: a Cochrane systematic review. Br J Anaesth. 2017;119:369-83.

45. Jiang J, Ma D, Li B, Yue Y, Xue F. Video laryngoscopy does not improve the intubation outcomes in emergency and critical patients - a systematic review and meta-analysis of randomized controlled trials. Crit Care. 2017:21:288.

46. Tisherman SA, Menaker J, Kon Z. Are we ready to take ECPR on the road? Maybe. Resuscitation. 2017;117:A1-2.

47. Nutbeam T. Clinical governance and prehospital care in the UK. Emerg Med J. 2011;28:91-2.

48. Ringdal KG, Lossius HM, Jones JM, Lauritsen JM, Coats TJ, Palmer CS, et al. Collecting core data in severely injured patients using a consensus trauma template: an international multicentre study. Crit Care. 2011;15:R237.

49. Jones J, Hunter D. Consensus methods for medical and health services research. BMJ. 1995;311:376-80

50. Angerman $\mathrm{S}$, Kirves $\mathrm{H}$, Nurmi J. A before-and-after observational study of a protocol for use of the C-MAC videolaryngoscope with a Frova introducer in pre-hospital rapid sequence intubation. Anaesthesia. 2018;73:348-55.

51. Anani N, Mazya MV, Chen R, Prazeres Moreira T, Bill O, Ahmed N, et al. Applying openEHR's guideline definition language to the SITS international stroke treatment registry: a European retrospective observational study. BMC medical informatics and decision making. 2017;17:7.

52. Rognas L, Hansen TM, Kirkegaard H, Tonnesen E. Predicting the lack of ROSC during pre-hospital CPR: should an end-tidal CO2 of $1.3 \mathrm{kPa}$ be used as a cut-off value? Resuscitation. 2014;85:332-5.

53. Chesters A, Keefe N, Mauger J, Lockey D. Prehospital anaesthesia performed in a rural and suburban air ambulance service staffed by a physician and paramedic: a 16-month review of practice. Emerg Med J. 2014;31:65-8.
54. Gellerfors M, Fevang E, Backman A, Kruger A, Mikkelsen S, Nurmi J, et al. Pre-hospital advanced airway management by anaesthetist and nurse anaesthetist critical care teams: a prospective observational study of 2028 pre-hospital tracheal intubations. Br J Anaesth. 2018;120:1103-9.

55. Lankimaki S, Alahuhta S, Silfvast T, Kurola J. Feasibility of LMA supreme for airway management in unconscious patients by ALS paramedics. Scand J Trauma Resusc Emerg Med. 2015;23:24

56. Lockey DJ, Avery P, Harris T, Davies GE, Lossius HM. A prospective study of physician pre-hospital anaesthesia in trauma patients: oesophageal intubation, gross airway contamination and the 'quick look' airway assessment. BMC Anesthesiol. 2013;13:21.

57. McQueen C, Crombie N, Hulme J, Cormack S, Hussain N, Ludwig F, et al Prehospital anaesthesia performed by physician/critical care paramedic teams in a major trauma network in the UK: a 12 month review of practice. Emerg Med J. 2015;32:65-9.

58. Nevin DG, Green SJ, Weaver AE, Lockey DJ. An observational study of paediatric pre-hospital intubation and anaesthesia in 1933 children attended by a physician-led, pre-hospital trauma service. Resuscitation. 2014;85:189-95.

59. Rhode MG, Vandborg MP, Bladt V, Rognas L. Video laryngoscopy in prehospital critical care - a quality improvement study. Scand J Trauma Resusc Emerg Med. 2016;24:84.

60. Rognas L, Hansen TM, Kirkegaard H, Tonnesen E. Pre-hospital advanced airway management by experienced anaesthesiologists: a prospective descriptive study. Scand J Trauma Resusc Emerg Med. 2013;21:58.

61. Rognas L, Hansen TM, Kirkegaard H, Tonnesen E. Refraining from prehospital advanced airway management: a prospective observational study of critical decision making in an anaesthesiologist-staffed pre-hospital critical care service. Scand J Trauma Resusc Emerg Med. 2013;21:75.

62. Rognas L, Hansen TM, Kirkegaard H, Tonnesen E. Standard operating procedure changed pre-hospital critical care anaesthesiologists' behaviour: a quality control study. Scand J Trauma Resusc Emerg Med. 2013;21:84.

63. Rognas L, Hansen TM, Kirkegaard H, Tonnesen E. Anaesthesiologist-provided prehospital airway management in patients with traumatic brain injury: an observational study. Eur J Emerg Med. 2014;21:418-23.

64. Raatiniemi L, Lankimaki S, Martikainen M. Pre-hospital airway management by non-physicians in northern Finland - a cross-sectional survey. Acta Anaesthesiol Scand. 2013:57:654-9.

65. Tarpgaard M, Hansen TM, Rognas L. Anaesthetist-provided pre-hospital advanced airway management in children: a descriptive study. Scand J Trauma Resusc Emerg Med. 2015;23:61.

66. Weatherall A, Garner A, Lovell N, Redmond S, Lee A, Skowno J, et al. Study protocol for the PHANTOM study: prehospital assessment of noninvasive tissue oximetry monitoring. Scand J Trauma Resusc Emerg Med. 2014;22:57.

67. Breckwoldt J, Klemstein S, Brunne B, Schnitzer L, Mochmann HC, Arntz HR Difficult prehospital endotracheal intubation - predisposing factors in a physician based EMS. Resuscitation. 2011:82:1519-24.

68. Burns BJ, Watterson JB, Ware S, Regan L, Reid C. Analysis of out-of-hospital pediatric intubation by an Australian helicopter emergency medical service. Ann Emerg Med. 2017;70:773-82 e4.

69. Crewdson K, Lockey D. Advanced airway management for pre-hospital trauma patients. Trauma. 2015;18:111-8.

70. de la Encina MEC, Quiles AS, Vicente-Mazariegos ID, Aracil NG, Alcaide LJ, Martinez MR. Efficacy and safety of endotracheal intubation performed in moving vs motionless environments. Emergencias. 2017:29:5-10.

71. Debacker M, Hubloue I, Dhondt E, Rockenschaub G, Ruter A, Codreanu T, et al. Utstein-style template for uniform data reporting of acute medical response in disasters. PLoS Curr. 2012;4:e4f6cf3e8df15a.

72. Fevang E, Lockey D, Thompson J, Lossius HM, Torpo Research C. The top five research priorities in physician-provided pre-hospital critical care: a consensus report from a European research collaboration. Scand J Trauma Resusc Emerg Med. 2011;19:57.

73. Fouche PF, Middleton PM, Zverinova KM. Training and experience are more important than the type of practitioner for intubation success. Crit Care. 2013;17:412

74. Fouche PF, Stein C, Simpson P, Carlson JN, Doi SA. Nonphysician out-ofhospital rapid sequence intubation success and adverse events: a systematic review and meta-analysis. Ann Emerg Med. 2017;70:449-59. e20

75. Fullerton JN, Roberts KJ, Wyse M. Should non-anaesthetists perform prehospital rapid sequence induction? An observational study. Emerg Med J. $2011 ; 28: 428-31$ 
76. Lockey D, Lossius HM. Pre-hospital airway management: the data grows rapidly but controversy remains. Resuscitation. 2014;85:849-50.

77. Prekker ME, Kwok H, Shin J, Carlbom D, Grabinsky A, Rea TD. The process of prehospital airway management: challenges and solutions during paramedic endotracheal intubation. Crit Care Med. 2014;42:1372-8.

78. Rehn M, Hyldmo PK, Magnusson V, Kurola J, Kongstad P, Rognas L, et al. Scandinavian SSAI clinical practice guideline on pre-hospital airway management. Acta Anaesthesiol Scand. 2016;60:852-64.

79. Rognas LK, Hansen TM. EMS-physicians' self reported airway management training and expertise; a descriptive study from the central region of Denmark. Scand J Trauma Resusc Emerg Med. 2011;19:10

80. Sollid SJ, Lossius HM, Nakstad AR, Aven T, Soreide E. Risk assessment of pre-hospital trauma airway management by anaesthesiologists using the predictive Bayesian approach. Scand J Trauma Resusc Emerg Med. 2010;18:22.

81. Sollid SJ, Lossius HM, Soreide E. Pre-hospital intubation by anaesthesiologists in patients with severe trauma: an audit of a Norwegian helicopter emergency medical service. Scand I Trauma Resusc Emerg Med. 2010;18:30.

82. Wigman LD, van Lieshout EM, de Ronde G, Patka P, Schipper IB. Traumarelated dispatch criteria for helicopter emergency medical Services in Europe. Injury. 2011;42:525-33.

83. Yeatts DJ, Dutton RP, Hu PF, Chang YW, Brown $\mathrm{CH}$, Chen $\mathrm{H}$, et al. Effect of video laryngoscopy on trauma patient survival: a randomized controlled trial. J Trauma Acute Care Surg. 2013;75:212-9.

84. Voigt R, Camp NP, Prabhakaran V, Hamilton WL, Hetey RC, Griffiths CM, et al. Language from police body camera footage shows racial disparities in officer respect. Proc Natl Acad Sci U S A. 2017;114:6521-6.

85. Fink A, Kosecoff J, Chassin M, Brook RH. Consensus methods: characteristics and guidelines for use. Am J Public Health. 1984;74:979-83.

Ready to submit your research? Choose BMC and benefit from:

- fast, convenient online submission

- thorough peer review by experienced researchers in your field

- rapid publication on acceptance

- support for research data, including large and complex data types

- gold Open Access which fosters wider collaboration and increased citations

- maximum visibility for your research: over $100 \mathrm{M}$ website views per year 